\author{
ĐẠI HỌC QUỐC GIA HÀ NỘI \\ TRƯỜNG ĐẠI HỌC KHOA HỌC Tự NHIÊN
}

ĐỒNG THỊ TRANG

\title{
MÔ HÌNH BLACK- SCHOLES CÓ TRỄ VÀ ỨNG DỤNG
}

LUẬN VĂN THẠC SỸ TOÁN HỌC

Hà Nội - 2014 


\title{
ĐẠI HỌC QUỐC GIA HÀ NÔ̂I
}

TRƯỜNG DẠI HỌC KHOA HỌC TỰ NHIÊN

ĐỒNG THỊ TRANG

\section{MÔ HİNH BLACK - SCHOLES CÓ TRẼ VÀ ỨNG DỤNG}

\author{
LUẬN VĂN THẠC SỸ TOÁN HỌC \\ Chuyên ngành: Lý thuyết xác suất và thống kê toán học \\ Mã số: 60460106
}

NGƯỜI HƯỚNG DÃ̃N KHOA HOC:

TS.LƯU HOÀNG ĐỨC

Hà Nội - 2014 


\section{Mục lục}

Lời mở đầu

1 Kiến thức chuẩn bị $\quad 5$

1.1 Quá trình ngẫu nhiên . . . . . . . . . . . . . . . . . 5

1.1 .1 Quá trình ngẫu nhiên . . . . . . . . . . . . . . . 5

1.1.2 Quá trình ngẫu nhiên thích nghi với bộ lọc . . . . . . . . . . . . 6

1.1.3 Kỳ vọng có điều kiện lấy đối với một $\sigma$-trường . . . . . . . . . . 6

1.1 .4 Xác suất có điều kiện . . . . . . . . . . . . . . . . . 7

1.1.5 Martingale . . . . . . . . . . . . . . 7

1.1.6 Quá trình Wiener hay chuyển động Brown . . . . . . . . . . . 8

1.1.7 Biến phân bậc hai của quá trình ngẫu nhiên . . . . . . . . . 9

1.1 .8 Tích phân Ito . . . . . . . . . . . . . . . . . . . . . . . 10

1.2 Phương trình vi phân ngẫu nhiên . . . . . . . . . . . . . . . . . . . . 13

1.2.1 Phương trình vi phân ngẫu nhiên thông thường . . . . . . . . . 13

1.2 .2 Công thức Ito . . . . . . . . . . . . . . . . . . . . 14

1.2.3 Phương trình vi phân ngẫu nhiên có trễ . . . . . . . . . . . . 16

1.3 Các khái niệm cơ bản trong tài chính . . . . . . . . . . . . . . . . . . 18

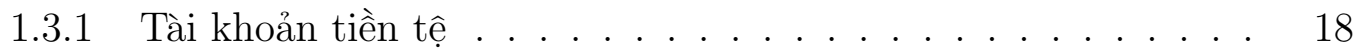

1.3.2 Thị trường tài chính . . . . . . . . . . . . . . . . . . . . . 18

1.3.3 Danh mục đầu tư tự tài trợ và không có độ chênh thị giá . . . 20

1.3.4 Chiến lược đáp ứng . . . . . . . . . . . . . . . . . . . 21

1.3.5 Xác suất trung hòa rủi ro . . . . . . . . . . . . . . 22

2 Mô hình Black - Scholes 24

2.1 Mô hình Black - Scholes cho phương trình vi phân ngẫu nhiên thông thường . . . . . . . . . . . . . . . . . . . . . . 24

2.1.1 Mô hình quá trình giá . . . . . . . . . . . . . . . . . . . . 24 
2.1.2 Mô hình Black - Scholes cho phương trình vi phân ngẫu nhiên thông thường . . . . . . . . . . . . . . . . 25

2.2 Mô hình Black - Scholes cho phương trình vi phân ngẫu nhiên có trễ . 29

2.2.1 Mô hình giá cổ phiếu có trễ . . . . . . . . . . . . . . . . . . 29

2.2.2 Mô hình Black -Scholes cho phương trình vi phân ngẫu nhiên có trễ hằng số . . . . . . . . . . . . . . . . . . . . . . . . 31

2.3 So sánh mô hình Black - Scholes và Black - Scholes có trễ . . . . . . . . 38

3 Ửng dụng của mô hình Black - Scholes có trễ xác định bong bóng hay sụp đổ trong thị trường tài chính 44

3.1 Mô hình hóa kinh tế . . . . . . . . . . . . . . . . . . . . . . . . . . . 44

3.2 Những tác động kinh tế . . . . . . . . . . . . . . . . . . . 51

3.3 Chiến lược đầu tư . . . . . . . . . . . . . . . . . . . . . . . 55

Kết luận $\quad 58$

Tài liệu tham khảo $\quad 59$ 


\section{Lời mở đầu}

Hiện nay, các mô hình ngẫu nhiên đã trở thành một trong những đối tượng nghiên cứu quan trọng trong lí thuyết toán tài chính, giúp chúng ta có công cụ để phân tích và định giá tài sản tài chính một cách tốt nhất. Công trình có tính chất cách mạng trong việc tính toán tài chính xuất hiện vào năm 1973 của F.Black và M.Scholes về tính giá trị hợp lý của các quyền chọn ("Pricing of Option and Corporate Liabilities"). Tiếp đó, có một loạt công trình về tính giá hợp lý của các quyền chọn và các sản phẩm tài chính với những mô hình ở nhiều cấp độ từ đơn giản đến phức tạp khác nhau, đáng chú ý là việc đưa ra mô hình Black - Scholes có trễ. Trong mô hình Black - Scholes có trễ, sự biến động trong quá khứ ảnh hưởng đến sự biến động hiện tại. Nó phù hợp với thị trường tài chính hơn so với mô hình Black - Scholes cổ điển.

Mục đích của luận văn là hệ thống lại một cách cơ bản mô hình Black - Scholes cho phương trình vi phân thông thường và phương trình vi phân có trễ, chỉ ra mối liên hệ cũng như sự khác nhau giữa hai mô hình này trong việc định giá quyền chọn. Luận văn cũng cung cấp các bài toán ứng dụng của mô hình Black - Scholes có trễ trong việc dự đoán khả năng xảy ra bong bóng và sụp đổ thị trường.

Bố cục luận văn bao gồm 3 chương:

- Chương 1 trình bày một số kiến thức chuẩn bị cần thiết, bao gồm các quá trình ngẫu nhiên, tích phân ngẫu nhiên, phương trình vi phân ngẫu nhiên thông thường, phương trình vi phân ngẫu nhiên có trễ, các khái niệm cơ bản về thị trường tài chính và cấu trúc của nó.

- Chương 2 là chương chính, trình bày mô hình Black - Scholes cho phương trình vi phân ngẫu nhiên thông thường và phương trình vi phân ngẫu nhiên có trễ, đồng thời xây dựng công thức định giá quyền chọn cho cả hai mô hình và so sánh mối liên hệ cũng như sự khác nhau giữa hai mô hình.

- Chương 3 trình bày việc ứng dụng của mô hình Black - Scholes có trễ trong việc xác định khả năng xảy ra bong bóng và sụp đổ thị trường. 
Luận văn được hoàn thành nhờ có sự hướng dẫn và giúp đỡ tận tình của Tiến sĩ Lưu Hoàng Đức, Viện Toán học - Viện Hàn lâm Khoa học và Công nghệ Việt Nam. Thầy đã dành nhiều thời gian hướng dẫn, giải đáp thắc mắc của em trong suốt quá trình làm luận văn. Qua đây, em xin được bày tỏ lòng biết ơn sâu sắc tới Thầy.

Cuối cùng em xin chân thành cảm ơn các thầy cô giáo giảng dạy tại trường Đại học Khoa học tự nhiên đã tận tình cung cấp kiến thức nền tảng cho em trong những năm học vừa qua.

Hà Nội, ngày 5 tháng 12 năm 2014

Học viên

\section{Đồng Thị Trang}




\section{Chương 1}

\section{Kiến thức chuẩn bị}

\subsection{Quá trình ngẫu nhiên}

Cho $(\Omega, \mathcal{F}, \mathbb{P})$ là một không gian xác suất, tức là một bộ ba gồm

1. $\Omega$ là một tập cơ sở bất kỳ nào đó mà mỗi phần tử $\omega \in \Omega$ đại diện cho một yếu tố ngẫu nhiên. Mỗi tập con của $\Omega$ gồm một số yếu tố ngẫu nhiên nào đó.

2. $\mathcal{F}$ là một họ nào đó các tập con của $\Omega$, chứa $\Omega$ và đóng đối với phép hợp đếm được và phép lấy phần bù; nói các khác $\mathcal{F}$ là một $\sigma$ - trường các tập con của $\Omega$. Mỗi tập hợp $A \in \mathcal{F}$ sẽ được gọi là một biến cố ngẫu nhiên.

3. $\mathbb{P}$ là một độ đo xác định trên không gian đo $(\Omega, \mathcal{F})$.

\subsubsection{Quá trình ngẫu nhiên}

1. Một quá trình ngẫu nhiên $\left(X_{t}, t \geq 0\right)$ là một hàm hai biến $X(t, \omega)$ xác định trên tích $\mathbb{R}^{+} \times \Omega$ lấy giá trị trong $\mathbb{R}$, và là một hàm đo được đối với $\sigma$ - trường tích $\mathcal{B}_{\mathbb{R}^{+}} \times \mathcal{F}$, trong đó $\mathcal{B}_{\mathbb{R}^{+}}$là $\sigma$ - trường các tập Borel trên $\mathbb{R}^{+}=[0, \infty)$. Điều đó có nghĩa là với mọi tập Borel $B$ trên $\mathbb{R}$ thì tập hợp

$$
\left\{(t, \omega) \in \mathbb{R}^{+} \times \Omega: X(t, \omega) \in B\right\}
$$

là một phần tử của $\sigma$ trường tích $\mathcal{B}_{\mathbb{R}^{+}} \times \mathcal{F} ; \sigma$ - trường này là $\sigma$ - trường nhỏ nhất chứa các tập có dạng

$$
[0, t] \times A, \quad \text { với } t \in \mathbb{R}^{+} \quad \text { và } A \in \mathcal{F} \text {. }
$$


2. Khi cố định một $\omega \in \Omega$ thì ánh xạ riêng phần $t \rightarrow X(t, \omega)$ từ $\mathbb{R}^{+}$vào $\mathbb{R}$ được gọi là một quỹ đạo của quá trình ngẫu nhiên $X=\left(X_{t}, t \geq 0\right)$ ứng với yếu tố ngẫu nhiên $\omega$ ấy.

\subsubsection{Quá trình ngẫu nhiên thích nghi với bộ lọc}

1. Một họ các $\sigma$ - trường con $\left(\mathcal{F}_{t}, t \geq 0\right)$ của $\mathcal{F}, \mathcal{F}_{t} \subset \mathcal{F}$ được gọi là bộ lọc thỏa mãn các điều kiện thông thường nếu

- Đó là họ tăng theo $t$, tức là $\mathcal{F}_{s} \subset \mathcal{F}_{t}$ nếu $s<t$;

- Họ đó là liên tục phải, tức là $\mathcal{F}_{t}=\bigcap_{\epsilon>0} \mathcal{F}_{t+\epsilon}$

- Nếu $A \in \mathcal{F}$ và $\mathbb{P}(A)=0$ thì $A \in \mathcal{F}_{0}$ (và do đó $A$ nằm trong mọi $\mathcal{F}_{t}$ )

2. Cho một quá trình ngẫu nhiên $X=\left(X_{t}, t \geq 0\right)$. Ta xét $\sigma$-trường $\mathcal{F}_{t}^{X} \sinh$ bởi tất cả các biến ngẫu nhiên $X_{s}$ với $s \leq t$ : $\mathcal{F}_{t}^{X}=\sigma\left(X_{s}, s \leq t\right)$. $\sigma$-trường này chứa đựng mọi thông tin về diễn biến quá khứ của quá trình $X$ cho đến thời điểm t. Người ta gọi đó là bộ lọc tự nhiên của quá trình $X$, hay lịch sử của $X$, hay cũng còn gọi là trường thông tin về $X$.

3. Một không gian xác suất $(\Omega, \mathcal{F}, \mathbb{P})$ trên đó ta gắn thêm vào một bộ lọc $\left(\mathcal{F}_{t}\right)$ được gọi là không gian xác suất được lọc và ký hiệu là $\left(\Omega, \mathcal{F},\left(\mathcal{F}_{t}\right), \mathbb{P}\right)$.

\subsubsection{Kỳ vọng có điều kiện lấy đối vởi một $\sigma$-trường}

1. Cho $(\Omega, \mathcal{F}, \mathbb{P})$ là một không gian xác suất, $\mathcal{G}$ là một $\sigma$ - trường con của $\mathcal{F}$ và $X$ là một biến ngẫu nhiên, tức là một ánh xạ đo được từ $(\Omega, \mathcal{F})$ vào $\left(\mathbb{R}, \mathcal{B}_{\mathbb{R}}\right)$, trong đó $\mathcal{B}_{\mathbb{R}}$ là $\sigma$-trường các tập Borel trên đường thằng $\mathbb{R}$.

Khi đó, một biến ngẫu nhiên $X^{*}$ sẽ được gọi là kỳ vọng có điều kiện của $X$ đối với $\sigma$-trường $\mathcal{G}$, nếu

- $X^{*}$ là biến ngẫu nhiên đo được đối với $\mathcal{G}$,

- Với mọi tập $A \in \mathcal{G}$ thì ta có $\int_{A} X^{*} d \mathbb{P}=\int_{A} X d \mathbb{P}$.

Biến ngẫu nhiên này sẽ được kí hiệu là $\mathbb{E}(X \mid G)$ và nó cũng là một biến ngẫu nhiên.

2. Nếu ta chọn $\sigma$-trường $\sigma(Y)$ sinh ra bởi một biến ngẫu nhiên $Y$ nào đó. Khi đó kỳ vọng có điều kiện của $X$ lấy đối với $\sigma(Y)$ cũng được ký hiệu là $\mathbb{E}(X \mid Y)$. 


\subsubsection{Xác suất có điều kiện}

Xác suất có điều kiện $\mathbb{P}(A \mid \mathcal{G})$ của một biến cố $A \in \mathcal{F}_{t}$ là biến ngẫu nhiên xác định bởi

$$
\mathbb{P}(A \mid \mathcal{G})=\mathbb{E}\left(\mathbb{1}_{A} \mid \mathcal{G}\right)
$$

trong đó $\mathbb{1}_{A}$ là hàm chỉ tiêu của một biến cố $A$, tức là

$$
\mathbb{1}_{A}(\omega)= \begin{cases}1 & \text { nếu } \omega \in A \\ 0 & \text { nếu } \omega \notin A\end{cases}
$$

\section{Tính chất}

1. $\mathbb{P}(\Omega \mid \mathcal{G})=1$ (hầu chắc chắn);

2. $\forall A \in \mathcal{F}: \mathbb{P}(\bar{A} \mid \mathcal{G})=1-\mathbb{P}(A \mid \mathcal{G})$ (hầu chắc chắn);

3. $\forall A_{1}, A_{2}, \cdots \in \mathcal{F}$ rời nhau từng đôi một thì

$$
\mathbb{P}\left(\bigcup_{n=1}^{\infty} A_{n} \mid \mathcal{G}\right)=\sum_{n=1}^{\infty} \mathbb{P}\left(A_{n} \mid \mathcal{G}\right) \quad \text { (hầu chắc chắn). }
$$

\subsubsection{Martingale}

Trong phần này chúng tôi trình bày các lý thuyết cơ bản về Martingale. Nội dung trình bày được trích dẫn từ tài liệu tham khảo [10, trang 200-201].

Định nghĩa 1.1. Cho không gian xác suất $(\Omega, \mathcal{F}, P)$ dược trang bị bộ lọc $\mathcal{F}=\left\{\mathcal{F}_{t}, 0 \leq\right.$ $t \leq T\}$. Một quá trình ngẫu nhiên thời gian liên tục $\{X(t)\}$ thích nghi với bộ lọc $\left\{\mathcal{F}_{t}\right\}$ được gọi là một martingale đối với $\mathcal{F}$ nếu thỏa mãn các điều kiện sau:

1. $E|X(t)|<\infty, \forall t \in[0, T]$,

2. $E\left[X(s) \mid \mathcal{F}_{t}\right]=X(t), \quad t<s \leq T$.

Giả sử s và t là hai giá trị bất kì sao cho $s \leq t$. Khi đó:

1. Nếu $\mathbb{E}\left(X_{t} \mid \mathcal{F}_{s}\right) \leq X_{s}$ thì $X$ gọi là martingale trên;

2. Nếu $\mathbb{E}\left(X_{t} \mid \mathcal{F}_{s}\right) \geq X_{s}$ thì $X$ gọi là martingale dưới.

Định nghĩa 1.2. Cho $\{X(t), 0 \leq t \leq T\}$ là một martingale. 
1. $\{X(t)\}$ được gọi là bình phương khả tích nếu

$$
\sup _{0 \leq t \leq T} E\left[X^{2}(t)\right]<\infty
$$

2. $\{X(t)\}$ được gọi là khả tích đều nếu

$$
\lim _{n \rightarrow \infty} \sup _{0 \leq t \leq T} E\left[|X(t)| \mathbb{1}_{\{|X(t)|>n\}}\right]=0 .
$$

Mệnh đề 1.1. 1. Mọi martingale bình phương khả tích xác dịnh trên khoảng thời gian hữu hạn là khả tích đều.

2. Cho Y là một biến ngẫu nhiên khả tích, định nghĩa

$$
M(t)=E\left[Y \mid \mathcal{F}_{t}\right], \quad 0 \leq t \leq T
$$

Khi đó, quá trình $\{M(t)\}$ khả tích đều.

Thời điểm ngẫu nhiên $\tau$ được gọi là thời điểm dừng nếu

$$
\{\tau \leq t\} \in \mathcal{F}_{t}, \quad \forall t \in[0, T]
$$

Một martingale bị dừng ở thời điểm $\tau$ là quá trình $\{M(t \wedge \tau), 0 \leq t \leq T\}$, trong đó $t \wedge \tau=\min \{t, \tau\}$. Nếu $\{M(t)\}$ là một martingale thì quá trình $\{M(t \wedge \tau)\}$ cũng là một martingale nhưng điều ngược lại thì không đúng.

Định nghĩa 1.3. (Martingale dịa phương) Một quá trình thích nghi $\{X(t)\}$ được gọi là một martingale địa phương nếu tồn tại một dãy các thời diểm dùng $\left\{\tau_{n}\right\}$ thỏa mãn $\tau_{n} \rightarrow \infty$ khi $n \rightarrow \infty$ và với mỗi $n$, quá trình $\left\{M\left(t \wedge \tau_{n}\right)\right\}$ là martingale khả tích đều. Dãy các thời điểm dùng $\left\{\tau_{n}\right\}$ dược gọi là dãy dịa phương hóa.

\subsubsection{Quá trình Wiener hay chuyển động Brown}

Trong phần này chúng tôi trình bày lý thuyết về chuyển động Brown, hàm xác suất chuyển của chuyển động Brown và đưa ra một số martingale quen thuộc tạo thành từ chuyền động Brown. Nội dung trình bày được trích dẫn từ tài liệu tham khảo [10, trang 46-47] và [3].

Một quá trình ngẫu nhiên $W=\left(W_{t}\right)_{t \geq 0}$ là một quá trình Wiener hay chuyển động Brown nếu:

1. $W_{0}=0$ hầu chắc chắn. 


\section{Tài liệu tham khảo}

1. Nguyễn Văn Hữu, Vương Quân Hoàng (2007), Các phương pháp toán học trong tài chính. Nhà xuất bản Đại học Quốc gia Hà Nội.

2. Trần Hùng Thao (2009), Nhập môn toán học tài chính. Nhà xuất bản Khoa học và Kỹ thuật.

3. Trần Hùng Thao (2013), Toán tài chính căn bản. Nhà xuất bản Văn hóa Thông tin.

4. Đặng Hùng Thắng (2006), Quá trình ngẫu nhiên và tính toán ngẫu nhiên. Nhà xuất bản Đại học Quốc gia Hà Nội.

5. Nguyễn Duy Tiến (2001), Các mô hình xác suất và úng dụng, Phần III: Giải tích ngẫu nhiên. Nhà xuất bản Đại học Quốc gia Hà Nội.

6. M. Arrioas, Y. Hu, S.E. Mohammed, and G. Pap (2007) A delayed Black and Scholes formula, Stochastic Analysis and Applications, 25(2), 471-492.

7. Appleby, J.A.D, Ridle, M. Swords (2012) Bubbles and crashes in a Black-Scholes Model with Delay, Finance Stochastics, 17(1), 1-30.

8. C.T.H. Baker, E. Buckwar (2000) Numerical analysis of explicit one-step methods for stochastic delay differential equations, Journal of Computation and Mathematics, 3, 315335.

9. M. Kijima (2003) Stochastic processes with applications to finance. Chapman \& Hall/CRC. 